\title{
Model-based Design of Experiments for Polyether Production from Bio-based 1,3-Propanediol
}

\author{
Anh-Duong Vo ${ }^{1}$, Ali Shahmohammadi ${ }^{2}$, and Kimberley McAuley ${ }^{1}$ \\ ${ }^{1}$ Queen's University \\ ${ }^{2}$ University of Texas at Austin
}

April 8, 2021

\begin{abstract}
Sequential model-based design of experiments (MBDOE) is used to select operating conditions for new experiments in a batchreactor that produces bio-based poly(trimethylene) ether glycol (PO3G). These Bayesian A-optimal experiments are designed to obtain improved estimates of the 70 fundamental-model parameter estimates, while accounting for the model structure and for data from eight previous industrial batch-reactor runs. Settings are selected for three decision variables: reactor temperature, initial catalyst level, and initial water concentration. If only one new experiment is conducted, it should be run at high temperature, with relatively high concentrations of catalyst and initial water. When two new runs are conducted, one should use an intermediate catalyst concentration. The effectiveness of the proposed MBDOE approach is tested using Monte-Carlo simulations, revealing that the selected experiments are superior compared to new experiments selected randomly from corners of the permissible design space.
\end{abstract}

\section{Hosted file}

P03G - MBDOE - Revised.pdf available at https://authorea.com/users/406673/articles/ 517260-model-based-design-of-experiments-for-polyether-production-from-bio-based-1-3propanediol 